\title{
Programming accessibility of DNA monolayers for degradation-free whole-blood biosensors
}

Mengying Deng ${ }^{\ddagger},,^{\dagger}, \perp, \mathrm{Min} \mathrm{Li}^{\dagger}, \stackrel{\perp}{ }, \mathrm{Fan} i^{\dagger}, \stackrel{\perp}{ }$, Xiuhai Mao ${ }^{\dagger}$, Qian $\mathrm{Li}^{\dagger}$, Jianlei Shen ${ }^{\dagger}$, Chunhai Fan ${ }^{*}{ }^{\dagger}$ and Xiaolei Zuo*, ${ }^{\dagger}$

\$Division of Physical Biology, CAS Key Laboratory of Interfacial Physics and Technology, Shanghai Synchrotron Radiation Facility (SSRF), Shanghai Institute of Applied Physics, Chinese Academy of Sciences, Shanghai 201800, China

§University of Chinese Academy of Science, Beijing 100049, China

$†$ School of Chemistry and Chemical Engineering, Institute of Molecular Medicine, Renji Hospital, School of Medicine, Shanghai Jiao Tong University, Shanghai 200240, China

$\perp$ These authors contributed equally.

*Corresponding author: fanchunhai@sjtu.edu.cn, zuoxiaolei@sjtu.edu.cn. 


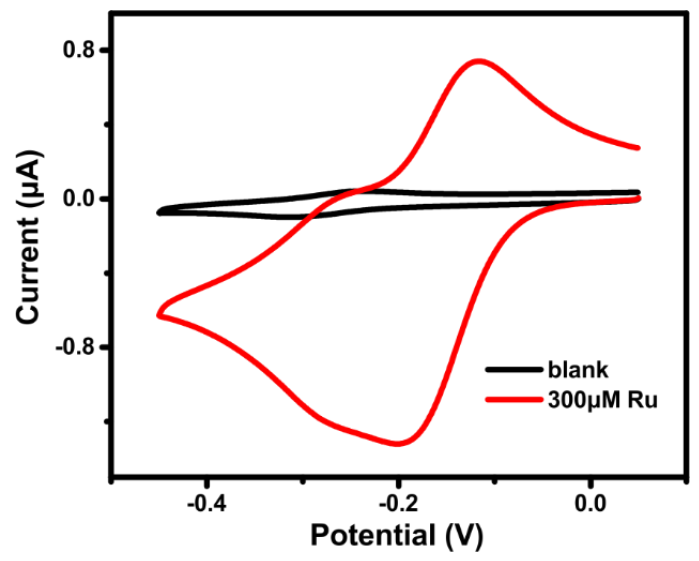

Figure S1. Cyclic voltammetry of DNA monolayer with and without $300 \mu \mathrm{M}$ of $\mathrm{RuHe}_{\mathrm{x}}$. 


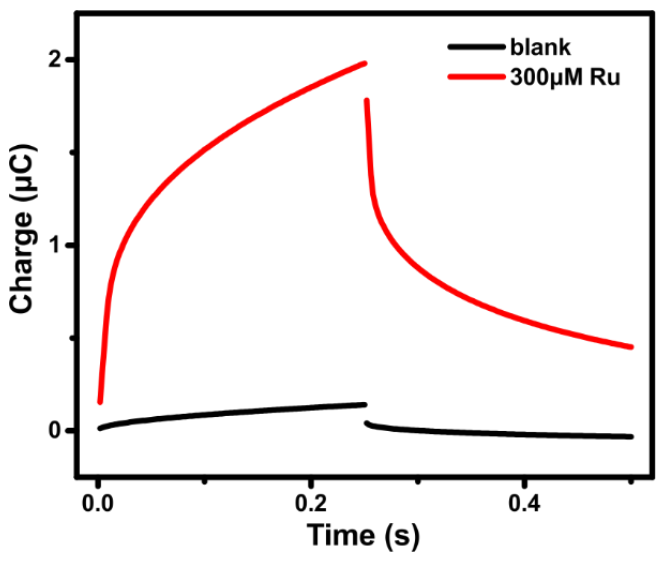

Figure S2. Chronocoulometry characterization of DNA monolayer with and without $300 \mu \mathrm{M}$ of $\mathrm{RuHe}_{\mathrm{x}}$. 


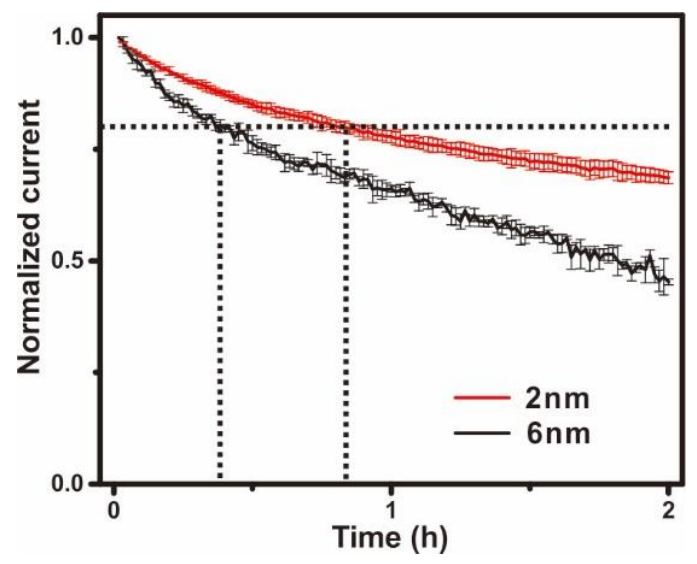

Figure S3. The stability of the DNA monolayers in the whole blood for 2 hours. 


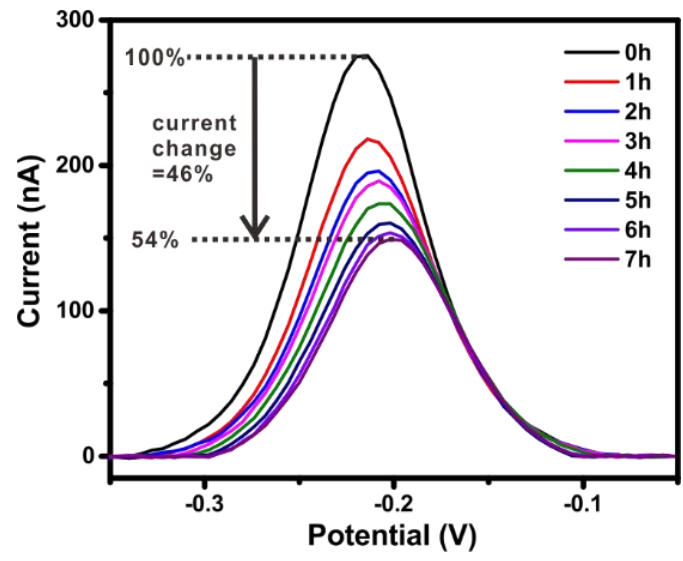

Figure S4. The current changes of DNA monolayer with lateral distance of $\sim 2 \mathrm{~nm}$ continuous scanning for 7 hours. 


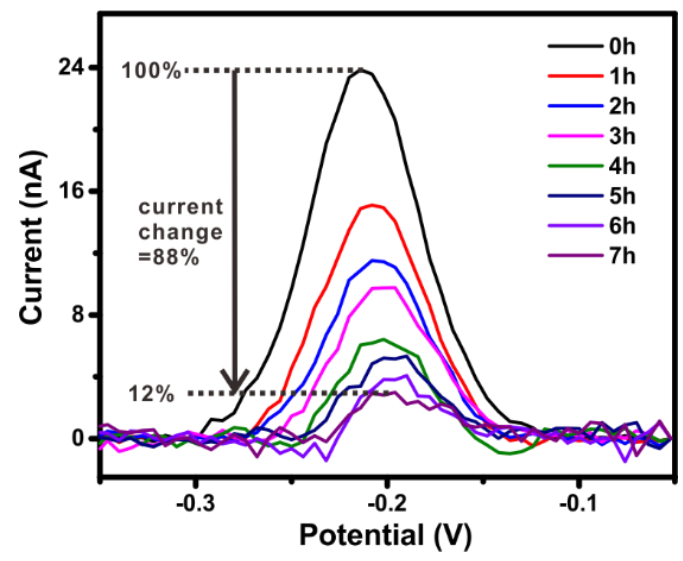

Figure S5. The current changes of DNA monolayer with lateral distance of $\sim 6 \mathrm{~nm}$ continuous scanning for 7 hours. 


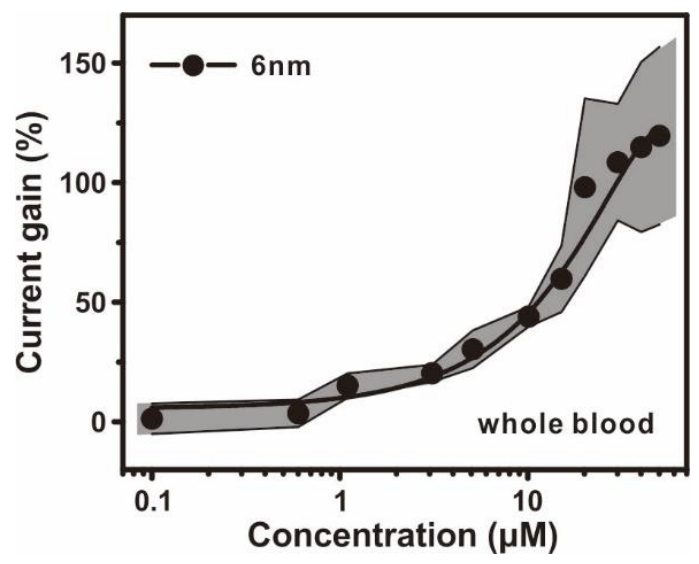

Figure S6. Titration curve for Dox detection in whole blood using DNA biosensor with lateral distance of $\sim 6 \mathrm{~nm}$. Each data point was an average of three independent experiment $(\mathrm{N}=3)$ and the error bars means the standard deviations. The fitted Kd was 15.11 $\mu \mathrm{M}$. 


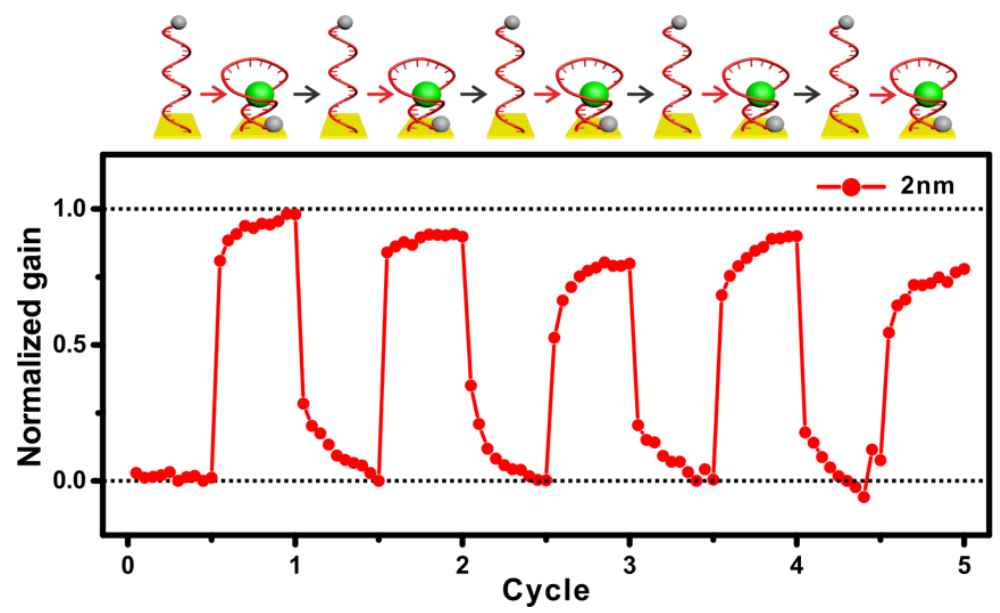

Figure S7. The reusability verification of DNA biosensor with lateral distance of $\sim 2 \mathrm{~nm}$ in fetal bovine serum by square wave voltammetry, the concentration of Dox was $5 \mu \mathrm{M}$. 


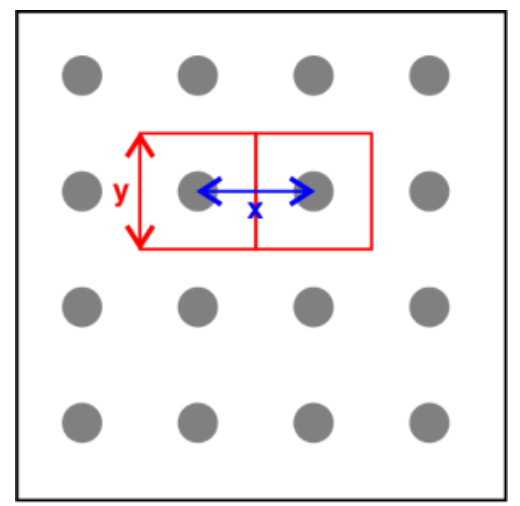

Figure S8. The schematic of lateral distance and surface density of DNA probe. The $\mathrm{x}$ is the lateral distance between two probes, and the $y$ is the side length of the square. 
Table S1. Comparisons of methods for Dox detection in complex matrices.

\begin{tabular}{|c|c|c|c|c|c|c|}
\hline Journal & $\mathbf{K d}(\boldsymbol{\mu} \mathbf{M})$ & $\begin{array}{c}\text { Dynamic } \\
\text { range }(\mu \mathrm{M})\end{array}$ & $\begin{array}{c}\text { Current drift before } \\
\text { processing }\end{array}$ & $\begin{array}{l}\text { Whole } \\
\text { blood }\end{array}$ & $\begin{array}{c}\text { External auxiliary } \\
\text { equipment }\end{array}$ & $\begin{array}{l}\text { Nanoscale } \\
\text { regulation }\end{array}$ \\
\hline $\begin{array}{l}\text { Sci. Transl. } \\
\text { Med. }(2013)^{1}\end{array}$ & $\sim 2$ & $0.01-10$ & $\sim 30 \%$ (3 hours) & Yes & $\begin{array}{l}\text { Continuous-flow } \\
\text { diffusion filter }\end{array}$ & $\mathrm{N} / \mathrm{A}$ \\
\hline $\begin{array}{l}\text { Int. J. Electro- } \\
\text { chem. Sci. } \\
(2016)^{2}\end{array}$ & $\mathrm{~N} / \mathrm{A}$ & $0.001-0.25$ & $\mathrm{~N} / \mathrm{A}$ & Yes & $\mathrm{N} / \mathrm{A}$ & Yes \\
\hline $\begin{array}{l}\text { J. Am. Chem. } \\
\text { Soc. }(2016)^{3}\end{array}$ & $\mathrm{~N} / \mathrm{A}$ & $\mathrm{N} / \mathrm{A}$ & $\sim 50 \%$ (8 hours) & Yes & $\mathrm{N} / \mathrm{A}$ & Yes \\
\hline $\begin{array}{c}\text { Mater. Sci. } \\
\text { Eng. C }(2017)^{4}\end{array}$ & $\mathrm{~N} / \mathrm{A}$ & $0.1-5.17$ & $\mathrm{~N} / \mathrm{A}$ & Yes & $\mathrm{N} / \mathrm{A}$ & Yes \\
\hline $\begin{array}{l}\text { Proc. Natl. } \\
\text { Acad. Sci. } \\
\text { U.S.A. }(2017)^{5}\end{array}$ & $\sim 5$ & $0.1-10$ & $\sim 40 \%$ (6 hours) & Yes & $\begin{array}{l}\text { Microporous }(0.2 \\
\mu \mathrm{m}) \text { polysulfone } \\
\text { membrane }\end{array}$ & $\mathrm{N} / \mathrm{A}$ \\
\hline $\begin{array}{l}\text { Nat. Biomed. } \\
\text { Eng. }(2017)^{6}\end{array}$ & $\sim 1.68$ & $0.05-8$ & $\mathrm{~N} / \mathrm{A}$ & Yes & Microfluidic device & $\mathrm{N} / \mathrm{A}$ \\
\hline $\begin{array}{l}\text { J. Am. Chem. } \\
\text { Soc. }(2017)^{7}\end{array}$ & $\sim 69.2$ & $4-100$ & $\mathrm{~N} / \mathrm{A}$ & Yes & $\mathrm{N} / \mathrm{A}$ & $\mathrm{N} / \mathrm{A}$ \\
\hline $\begin{array}{l}\text { Angew. Chem. } \\
\text { Int. Ed. }(2017)^{8}\end{array}$ & $\sim 100$ & $10-200$ & $\begin{array}{c}\sim 10 \% \text { (12 hours) with } \\
\text { phosphatidylcholine } \\
\sim 70 \% \text { (12 hours) with } \\
\text { 6-mercapto-1-hexanol } \\
\sim 50 \% \text { (12 hours) with } \\
\text { 11-mercapto-1-un- } \\
\text { decanol }\end{array}$ & Yes & $\mathrm{N} / \mathrm{A}$ & Yes \\
\hline $\begin{array}{l}\text { This work } \\
\text { (2019) }\end{array}$ & $\sim 7.75$ & $0.1-40$ & $\begin{array}{l}31.35 \% \text { ( } 3 \text { hours) } \\
44.29 \% \text { ( } 6 \text { hours) }\end{array}$ & Yes & $\mathrm{N} / \mathrm{A}$ & Yes \\
\hline
\end{tabular}

\section{References}

(1) Ferguson, B. S.; Hoggarth, D. A.; Maliniak, D.; Ploense, K.; White, R. J.; Woodward, N.; Hsieh, K.; Bonham, A. J.; Eisenstein, M.; Kippin, T. E.; Plaxco, K. W.; Soh, H. T. Real-Time, Aptamer-Based Tracking of Circulating Therapeutic Agents in Living Animals. Sci. Transl. Med. 2013, 5, 213ra165-213ra165.

(2) Peng, A.; Xu, H.; Luo, C.; Ding, H. J. I. J. E. S. Application of A Disposable Doxorubicin Sensor for Direct Determination of Clinical Drug Concentration in Patient Blood. Int. J. Electrochem. Sci. 2016, 11, 6266-6278.

(3) Li, H.; Arroyo-Currás, N.; Kang, D.; Ricci, F.; Plaxco, K. W. Dual-Reporter Drift Correction to Enhance the Performance of Electrochemical Aptamer-Based Sensors in Whole Blood. J. Am. Chem. Soc. 2016, 138, 15809-15812.

(4) Soleymani, J.; Hasanzadeh, M.; Eskandani, M.; Khoubnasabjafari, M.; Shadjou, N.; Jouyban, A. Electrochemical Sensing of Doxorubicin in Unprocessed Whole Blood, Cell Lysate, and Human Plasma Samples Using Thin Film of Poly-Arginine Modified Glassy Carbon Electrode. Mater. Sci. Eng. C 2017, 77, 790-802.

(5) Arroyo-Curras, N.; Somerson, J.; Vieira, P. A.; Ploense, K. L.; Kippin, T. E.; Plaxco, K. W. Real-Time Measurement of Small Molecules Directly in Awake, Ambulatory Animals. Proc. Natl. Acad. Sci. U.S.A. 2017, 114, 645-650.

(6) Mage, P. L.; Ferguson, B. S.; Maliniak, D.; Ploense, K. L.; Kippin, T. E.; Soh, H. T. Closed-Loop Control of Circulating Drug Levels in Live Animals. Nat. Biomed. Eng. 2017, 1, 0070.

(7) Li, H.; Dauphin-Ducharme, P.; Ortega, G.; Plaxco, K. W. Calibration-Free Electrochemical Biosensors Supporting Accurate Molecular Measurements Directly in Undiluted Whole Blood. J. Am. Chem. Soc. 2017, 139, 11207-11213.

(8) Li, H.; Dauphin-Ducharme, P.; Arroyo-Currás, N.; Tran, C. H.; Vieira, P. A.; Li, S.; Shin, C.; Somerson, J.; Kippin, T. E.; Plaxco, K. W. A Biomimetic Phosphatidylcholine-Terminated Monolayer Greatly Improves the in Vivo Performance of Electrochemical Aptamer-Based Sensors. Angew. Chem. Int. Ed. 2017, 56, 7492-7495. 